SLAC-PUB-8215

August 1999

\title{
Predicting Induced Radioactivity at High Energy Accelerators
}

A. Fasso et al.

Ninth International Conference on Radiation Shielding, Tsukuba, Japan, October 17-22, 1999 


\title{
Predicting Induced Radioactivity at High-Energy Electron Accelerators
}

\author{
Alberto FASSÒ *, Marco SILARI ${ }^{\$ ; \dagger}$ and Luisa ULRICI ${ }^{\$}$ \\ * SLAC, Stanford Linear Accelerator Center \\ ${ }^{\$}$ CERN, European Organisation for Nuclear Research
}

\begin{abstract}
Radioactive nuclides are produced at high-energy electron accelerators by different kinds of particle interactions with accelerator components and shielding structures. Radioactivity can also be induced in air, cooling fluids, soil and groundwater. The physical reactions involved include spallations due to the hadronic component of electromagnetic showers, photonuclear reactions by intermediate energy photons and low-energy neutron capture. Although the amount of induced radioactivity is less important than that of proton accelerators by about two orders of magnitude, reliable methods to predict induced radioactivity distributions are essential in order to assess the environmental impact of a facility and to plan its decommissioning. Conventional techniques used so far are reviewed, and a newintegrated approach is presented, based on an extension of methods used at proton accelerators and on the unique capability of the FLUKA Monte Carlo code to handle the whole joint electromagnetic and hadronic cascade, scoring residual nuclei produced by all relevant particles. The radiation aspects related to the operation of superconducting RF cavities are also addressed.
\end{abstract}

\section{KEYWORDS: Monte-Carlo calculations, induced radioactivity, electron accelerator, photonuclear reactions, superconducting RF cavity.}

\section{Introduction}

Radioactivity induced by particle nuclear interactions in beamline elements and shielding structures represents one of the main radiation hazards of high-energy accelerators. Typically, the radioactivity levels at electron accelerators are lower than those found at proton accelerators, but the health physics issues are common to both: exposure of personnel, impact on the environment, disposal of material and decommissioning. But because not all physical interactions leading to induced radioactivity are the same, historically different techniques have been developed to predict activation at the two types of facility.

\section{Occupational exposure}

At most accelerator facilities the largest contribution to personnel dose arises from maintenance work near dumps, targets, septa, collimators and generally near any object hit directly by the primary beam or located close to a beam loss point. External or internal exposure to radiation from induced radioactivity can also occur in connection with handling, transport, machining, welding, chemical treatment and storage of irradiated items.

Secondary radiation generated by beam losses can also activate air, water and other fluids. Although most of the produced radioactive nuclides are short-lived, they can contribute to personnel dose if the accelerator enclosure is accessed during the first hour after shutdown or if the fluid is allowed to circulate outside the shielding during operation.

${ }^{\dagger}$ Corresponding author,

Tel +41-22-7673937; Fax +41-22-7675700

E-mail: marco.silari@cern.ch

* Radiation Physics Department, P.O. Box 4349, Stanford, CA

94309, USA.

\$1211 Geneva 23, Switzerland.
Work in a radioactive environment is an operational radiation protection issue which is addressed mainly by measurement and work planning. However, exposure of personnel can be minimized by good engineering design, provided reliable tools or techniques are available to predict activation of various accelerator parts.

\section{Environmental impact}

Release of activated air and water, as well as activation of soil and groundwater by neutrons and other secondary particles, have also an environmental impact possibly extending beyond the boundary of the accelerator site. At electron accelerators, the radioactivity levels are generally low and in some cases hard to measure: however sound predicting techniques are essential in order to draw up an impact report (or equivalent document) as required by most national regulations.

\section{Disposal and decommissioning}

Accelerator decommissioning and more in general the disposal of activated materials presents a particular challenge: even very low levels of induced radioactivity must be assessed, and not only in a global way, but for each individual accelerator part that must be disposed of. This is a subject that has grown in importance only in recent years: while there is a rich literature concerning occupational and environmental issues in connection with induced radioactivity at high-energy accelerators, little can be found concerning radioactivity predictions in view of decommissioning. As a matter of fact, accelerator dismantling was generally handled in the past by setting up a plan of systematic measurements after the machine was shutdown ${ }^{(1,2)}$, but modern regulations require that decommissioning be planned for even as the facility is being designed and built ${ }^{(3)}$. In some cases, a special study including detailed calculations is requested, as for $\operatorname{LEP}^{(4)}$. 


\section{Physical interactions}

Radioactivity at electron accelerators is induced directly by photonuclear reactions or indirectly by secondary particles (neutrons, protons, pions) originated in the same reactions. Most of the radioactivity is directly induced by photons and is confined within a limited region of space, because the nuclear cross section is only a very small fraction of the total photon cross section and photon penetration in matter is governed only by electromagnetic interactions (Compton minimum). The most penetrating photons have mean free paths of the order of a few $\mathrm{cm}$ in metallic structures and about $20 \mathrm{~cm}$ in concrete.

On the other hand, the radioactivity induced by secondaries is distributed in space according to their own penetrating power. Secondary particles of different type and energy can be generated by photonuclear effects taking place at different photon energies.

\section{Giant Resonance}

Giant Dipole Resonance (GDR) interactions dominate at energies between threshold $(5-10 \mathrm{MeV})$ and about $30 \mathrm{MeV}$. In this energy range the cross-section is large (up to several hundred millibarn) and there is a large number of photons. Indeed, the energy is of the order of the critical energy of most materials, which is typical of photons at the maximum of an electromagnetic shower. In many cases, therefore, direct GDR activation is concentrated in a limited region of space around shower maximum, not far from the beam loss point.

At electron machines of very high energy, such as LEP, photons in the upper tail of the synchrotron radiation spectrum can also induce activity by GDR interactions. These too are contained in a narrow region surrounding the vacuum chamber.

GDR interactions also produce neutrons with energies of the order of $1 \mathrm{MeV}$, which can in turn interact with nuclei and contribute to induced radioactivity. The mean attenuation path of such neutrons is of the order of $10 \mathrm{~cm}$ in concrete, so the activity induced by them is roughly contained in the same region as that due to the parent photons. The photon fluence is much larger than that of neutrons, but neutrons have larger cross sections, especially when they become thermalized. Hence, both components must be taken into account.

\section{Quasi-deuteron effect}

In the energy range between 30 and few hundred $\mathrm{MeV}$, photons interact mainly via the quasi-deuteron effect. Photons in this energy range are less numerous, and the quasi-deuteron cross section is small compared to the GDR one. Therefore direct activation by such photons is also less important; however, their secondaries (mainly neutrons) have multiplicities and energies which increase with photon energy, and their activating effect may extend over a wider region of space.

\section{Delta Resonance}

At energies between $200 \mathrm{MeV}$ and a few $\mathrm{GeV}$, characterized by the Delta Resonance, pion production becomes superposed to the quasi-deuteron effect. The Delta Resonance cross section is rather large (of the order of one half of the GDR), but photons in this energy range are much less than at shower maximum, so their contribution to total activation is comparably small. However, nuclides directly produced in this type of reaction may dominate in thin targets where the electromagnetic shower is not fully developed. On the other hand, some of the secondary pions and neutrons may have sufficient energy to initiate a hadronic cascade extending well beyond the GDR activation region.

\section{High-energy photons}

Photons in the highest energy range, above the delta resonance, are created in the first beam interaction or at most in the first few generations of the ensuing electromagnetic shower (often within a fraction of a radiation length). They are few and their photonuclear cross section is small, and therefore their direct contribution to activation is negligible. However, they can originate hadronic cascades with a large number of secondary particles which can penetrate deep into the accelerator shielding, also activating soil and groundwater.

\section{Relative importance of the various contributions}

Since only one or few nucleons are removed, in a given material there are only a small number of radioactive nuclides which can be produced by direct GDR interaction. In some materials, such as lead, very little activity is produced. The most common radionuclides produced by GDR in accelerator structures are ${ }^{53} \mathrm{Fe},{ }^{55} \mathrm{Fe}$ and ${ }^{64} \mathrm{Cu}$.

Compilations of GDR cross sections are available ${ }^{(5,6)}$ but data are scarce for some important materials (iron). Some parameterizations have been proposed ${ }^{(5)}$ but only for medium and heavy nuclei.

The activation originated, directly or indirectly, by photons of energy higher than the GDR is similar in quality, if not in quantity, to that produced by high-energy hadrons. Globally, it does not depend strongly on specific materials, but varies rather smoothly with mass number so that in a given element, practically all radioactive nuclides with lower mass number can be produced by spallation or evaporation. The total cross sections are smooth functions of $\mathrm{A}$ and $\mathrm{Z}$ for energies above the GDR. However, the actual neutron multiplicity and the probability of producing a specific residual nucleus are not easily predicted without simulating the interaction in detail by means of a specialized code. A formula similar to that developed by Rudstam ${ }^{(7)}$ for highenergy protons has been proposed by Jonsson and Lindgren $^{(8)}$. Typical radionuclides produced in this energy range are ${ }^{3} \mathrm{H},{ }^{18} \mathrm{~F},{ }^{22} \mathrm{Na},{ }^{24} \mathrm{Na},{ }^{46} \mathrm{Sc},{ }^{47} \mathrm{Sc},{ }^{48} \mathrm{Sc},{ }^{51} \mathrm{Cr},{ }^{52} \mathrm{Mn}$ and ${ }^{54} \mathrm{Mn}$.

Direct activation by GDR $(\gamma, \mathrm{n})$ reactions is an important source of radioactivity in air and water ${ }^{(9,10)}$, while spallation contributes only to a limited extent. However, with the exception of ${ }^{11} \mathrm{C}$, which has a half life of about 20 minutes, most of the activity generated by GDR reactions is shortlived $\left({ }^{13} \mathrm{~N}\right.$ and $\left.{ }^{15} \mathrm{O}\right)$ and can only be of concern in the immediate surroundings of the accelerator. In air, the contribution to activation with the largest environmental 
impact comes from thermal neutrons, which produce the radionuclide with the longest half-life $\left({ }^{41} \mathrm{Ar}, 1.83\right.$ hours).

Because the total activity due to the hadronic cascade generated by high-energy photons is a small fraction of the total, that component is often considered to be of secondary importance. However, it is practically the only component outside thick shields and any environmental impact study must use some technique to evaluate its effect on soil and groundwater.

A particular case is that of activation by thermal neutrons. Although their fluence is generally small, they can induce non negligible activities in some nuclides of very high cross section present even in small amounts in concrete, soil or other materials. Typical examples are sodium in concrete, antimony in lead and several rare earths in soil. These activities are difficult to predict because the chemical composition of such materials is not known with sufficient accuracy, and in some cases (pure beta emitters) they are also hard to measure.

\section{Electron and proton accelerators}

Thirty years ago, DeStaebler ${ }^{(11)}$ estimated the dose rate due to induced radioactivity in the SLAC linear accelerator using the scarce data available at that time and much physical insight. Assuming that roughly $1 \%$ of the energy lost by a high-energy electron is being spent in nuclear interactions (based on the known ratio between the strength of nuclear and electromagnetic forces) and that each interaction absorbs on average $50 \mathrm{MeV}$, with the help of some classical shower theory and a few other considerations about the average properties of radioactive nuclei, he was able to predict the order of magnitude of the dose rate from induced radioactivity produced per unit power of electron beam.

Since then, various authors ${ }^{(10,12)}$ have confirmed that the total induced radioactivity is nearly proportional to beam power at both proton and electron accelerators, with a ratio of about two orders of magnitude between the two proportionality factors. However, due to the different spatial distribution of radioactivity in the two types of accelerators, this ratio cannot be used to scale specific activities and dose rate levels from proton to electron accelerators. The ratio applies only to induced radioactivity in air, water and unshielded accelerator structures, while the ratio outside thick shielding is probably much larger. But the present regulatory trend is such that even when extremely low radioactivity levels are expected, a full study must be carried out and detailed proof of the estimated activities must be reported anyway. Therefore, reliable methods to predict induced radioactivity distributions are essential in order to assess the environmental impact of an electron accelerator and to plan its decommissioning.

\section{Induced radioactivity in superconducting RF cavities}

A less conventional radiation source in present highenergy electron accelerators is represented by the superconducting radiofrequency (RF) cavities. These components are often an important radiation source both during conditioning without beam and during normal operation in the accelerator. Although this type of equipment is also used in proton accelerators, it is worth mentioning that in high-energy circular electron accelerators the RF system serves the double purpose of ramping the beam from the injection energy to the energy required for physics, and to compensate for the continuous energy loss due to synchrotron radiation during coasting. In fact most of the RF power is needed for this second task (which is not the case with proton accelerators), as the energy lost by synchrotron radiation increases with the fourth power of the beam energy. The LEP superconducting RF cavities operate, on average, at a gradient of $6 \mathrm{MV} / \mathrm{m}$, but in case of need they can be rapidly driven to their maximum, which can be as high as $9 \mathrm{MV} / \mathrm{m}$. Before being installed in the machine the cavities are actually conditioned to their maximum achievable field in shielded test areas.

The production of radiation is mainly caused by field emission of electrons from small impurities on the $\mathrm{Nb}$ surface. Electron multipacting (a resonance phenomenon linked to the electric surface field, the secondary electron emission coefficient of the surface, the geometry of the cavity and the frequency) is a less important mechanism if the $\mathrm{Nb}$ surface is properly cleaned ${ }^{(13)}$. Trajectory calculations have shown that there are essentially two electrons sources $^{(14,15)}$ : 1) a high intensity source of low energy electrons mainly producing transverse bremsstrahlung Xrays; and 2) a low intensity source of high energy electrons travelling parallel to the cavity axis and generating X-rays emitted along the axis. The latter source is more important, due to the much higher energy to which electrons can be accelerated to, up to the maximum field of the cavity. When these electrons strike the cavity walls or any other material, they produce intense bremsstrahlung radiation. Part of this bremsstrahlung radiation is sufficiently energetic to induce photonuclear reactions resulting in neutron production and activation of material.

In practice, a superconducting RF cavity can be regarded as a small electron linear accelerator: activation phenomena are therefore similar as they are governed by the same mechanisms. However, a peculiar aspect with which one is confronted when dealing with a large number of units, is that each cavity has its own "history" and the conditioning process can vary significantly from one cavity to the other. Therefore it is not easy to predict neither the intensity of the emitted radiation nor the expected amount of radioactivity induced in the surrounding structures. To cope with this lack of predictability, photon and neutron measurements were performed at CERN on several superconducting units (both single cavities and 4-cavity modules) during their conditioning before installation in $\mathrm{LEP}^{(16)}$. The measurements have shown a sharp increase in the radiation emission when the electric field is raised from about $6 \mathrm{MV} / \mathrm{m}$ to about $8.5 \mathrm{MV} / \mathrm{m}$ (Fig. 1), followed by a slow decrease as conditioning proceeded.

Although each cavity had already been conditioned at least once before being assembled with three other units in a module, the dose rates measured at the extremities of a module are much higher than for a single cavity, as the total 
field is a factor of 4 higher. For the same reason the induced radioactivity also increases with electric field. In addition, it was found that the dose rates at the two ends of a module are usually different, in some cases by as much as two orders of magnitude.

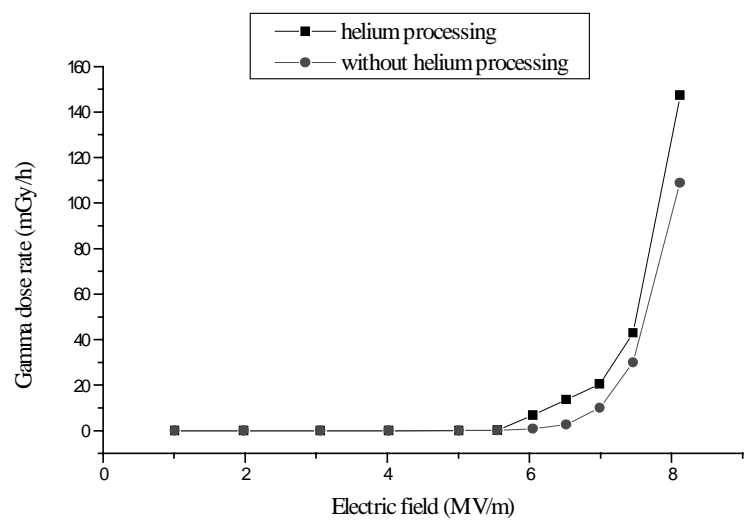

Fig. 1 Photon dose rate as a function of applied electric field measured at the extremity of a LEP superconducting RF single cavity, with and without helium processing (RF conditioning in an helium gas).

The components undergoing activation are essentially the extremities of the module and, during operation in LEP, the downstream components in the beam line. The residual dose rate at $10 \mathrm{~cm}$ distance from the activated component may reach several $\mathrm{mSv} / \mathrm{h}$ a few minutes after the module has been switched off. It was found that the dose rate decreases by about a factor of 10 in 40 minutes, due to the decay of short-lived radionuclides, followed by a much slower decrease (another factor of 10 in about 48 hours). The production reactions of the short-lived (less than one hour) radionuclides responsible for most of the induced radioactivity in stainless steel and copper (the materials most likely to be irradiated) in the first few minutes after the RF has been switched off can be supposed from the material composition. In stainless steel, the activity would mainly come from ${ }^{50} \mathrm{Cr}(\gamma, \mathrm{n}){ }^{49} \mathrm{Cr}$ (half-life $42.1 \mathrm{~min}$ ), ${ }^{54} \mathrm{Fe}(\gamma, \mathrm{n}){ }^{53} \mathrm{Fe}$ (8.51 min), ${ }^{54} \mathrm{Fe}(\gamma, \mathrm{n}){ }^{53 \mathrm{~m}} \mathrm{Fe} \quad(2.6 \mathrm{~min}),{ }^{92} \mathrm{Mo}(\gamma, \mathrm{n}){ }^{91 \mathrm{~m}} \mathrm{Mo}$ (1.09 min) and ${ }^{92} \mathrm{Mo}(\gamma, \mathrm{n}){ }^{91} \mathrm{Mo}(15.49 \mathrm{~min})$; in copper, from the two reactions ${ }^{63} \mathrm{Cu}(\gamma, n){ }^{62} \mathrm{Cu}$ (half-life $9.74 \mathrm{~min}$ ) and ${ }^{63} \mathrm{Cu}(\gamma, 3 \mathrm{n}){ }^{60} \mathrm{Cu}(23.2 \mathrm{~min})$.

Gamma spectrometry measurements of a copper absorber and a stainless steel flange carried out several days after irradiation have identified several long-lived radionuclides: ${ }^{51} \mathrm{Cr},{ }^{54} \mathrm{Mn},{ }^{56} \mathrm{Co},{ }^{57} \mathrm{Co},{ }^{58} \mathrm{Co},{ }^{60} \mathrm{Co},{ }^{65} \mathrm{Zn},{ }^{72} \mathrm{Se}$, ${ }^{75} \mathrm{Se},{ }^{74} \mathrm{As},{ }^{120} \mathrm{Sb}$ in copper; ${ }^{48} \mathrm{~V},{ }^{51} \mathrm{Cr},{ }^{52} \mathrm{Mn},{ }^{54} \mathrm{Mn},{ }^{56} \mathrm{Ni},{ }^{57} \mathrm{Ni}$, ${ }^{56} \mathrm{Co},{ }^{57} \mathrm{Co},{ }^{58} \mathrm{Co},{ }^{60} \mathrm{Co},{ }^{88} \mathrm{Y},{ }^{92 \mathrm{~m}} \mathrm{Nb},{ }^{95} \mathrm{Nb},{ }^{99} \mathrm{Mo}$ in stainless steel. The heaviest radionuclides found in stainless steel are most likely produced on impurities. In one case the thermic insulation was severely damaged by radiation and fell into pieces when the module was opened for maintenance. The insulation is constituted of several films of polyethylene terephthalate (PET) on which a thin aluminium layer (400 A) is deposited separated by a thick insulating layer made of polyester. A gamma-spectrometry analysis carried out on a sample taken from the damaged insulation showed traces of
${ }^{22} \mathrm{Na},{ }^{46} \mathrm{Sc},{ }^{51} \mathrm{Cr},{ }^{54} \mathrm{Mn}$ and ${ }^{65} \mathrm{Zn}$, most likely produced on impurities in the aluminium.

\section{Commonly adopted evaluation techniques}

In order to simulate activation in an electron accelerator environment, a Monte Carlo code must be able to handle not only both the electromagnetic and the hadronic cascade, but also the interaction between them, represented by photonuclear interactions. Since no such code was available until recent times, calculation techniques have been based so far mainly on a mix of analytical formulae, order of magnitude estimates and extrapolations from published measurements. In some rare cases ${ }^{(17,18)}$, specialized electromagnetic and/or hadronic codes have been used, but only to provide numerical values for the quantities appearing in analytical expressions.

\section{Calculating induced radioactivity from a source term}

Some authors ${ }^{(9,19,20,21)}$ have predicted the production of radioactivity in soil and groundwater in a straightforward way, by deriving neutron and hadron fluence at a point using a source term and the inverse square law, and multiplying the fluence value by average cross sections. In some cases ${ }^{(20,21)}$ a published dose attenuation formula and a dose to fluence conversion factor have been applied to account for the effect of shielding. This approach, although very simple, has been shown to give results close to those obtained by other techniques $^{(9,21)}$.

\section{Track-length calculations}

A more refined type of calculation is based on the total photon track-length (possibly differential) in a given volume. The track-length is obtained by ray tracing and assuming a given photon angular distribution or, in more modern studies, by a Monte Carlo photon transport code ${ }^{(22,23)}$. Provided production cross sections for each radionuclide of interest are available, this method gives accurate results, but only for nuclides directly produced in photon interactions and excluding activity induced by secondaries (GDR neutrons, high-energy hadrons).

The method is laborious, because good compilations of experimental photonuclear cross section are scarce and incomplete $^{(24)}$, but is the only mean to evaluate induced radioactivity originating from trace elements. Each cross section not found in the literature must be obtained by models or parameterizations ${ }^{(5,8)}$ having an uncertain range of validity. Indeed, while the value of the total inelastic cross section is a smooth function of mass number, competition between different reaction channels makes any partial cross section much more dependent on the individual properties of each target/product nucleus combination.

However, there are some cases where activation by secondaries is negligible and the cross sections needed are few and well known. The track-length technique is widely used for air activation calculations ${ }^{(9,22,23,25)}$ and has been used successfully at CERN to predict activation by hard 
synchrotron radiation in $\mathrm{LEP}^{(26)}$. This topic is addressed in another contribution of this conference ${ }^{(4)}$.

\section{Scaling with absorbed power}

The original approach of DeStaebler, mentioned in section III, supported by new available experimental data, was later improved by Swanson ${ }^{(27)}$ and has become the basis of a standard technique for evaluating the radioactivity induced by electron beams ${ }^{(9,23,25,28)}$. The total saturation activity of various radionuclides induced per unit power in an infinite slab has been estimated by Swanson, for a number of common materials, by multiplying the total photon tracklength (in Approximation A or B of Rossi's shower theory ${ }^{(29,30)}$ ) with the production cross section weighted by the inverse energy squared and integrated over all energies $^{(31)}$. The corresponding activity induced in any volume of a given material is scaled according to the energy deposited in that volume per unit time. The deposited energy is estimated as an order of magnitude, or by an electronphoton Monte Carlo code such as $\mathrm{EGS}^{(32)}$, especially in the most recent studies.

This approach, although widely adopted, is affected by many uncertainties. Experimental cross sections do not always exist, and even when they do, the measurements cover only a limited energy range: the gaps must be filled by extrapolation and guessing. The list of radioactive products with some importance for radiation protection reported in Swanson's book ${ }^{(27)}$ is not exhaustive, as it can be shown for instance by comparing with the experimental results of Sato et $a l{ }^{(33)}$. But most important, scaling with deposited power assumes implicitly that the photon spectrum, and especially the neutron spectrum, is identical everywhere: a condition far from being fulfilled, as it has been pointed out before. A further source of error comes from ignoring the fact that in most cases the electromagnetic shower does not develop in a single, semi-infinite material, but in complex multi-material structures. In such conditions, it is not obvious that simple analytical shower theory approximations be able to accurately predict photon spectra. Swanson claims that his predictions for an infinite slab should be valid within a factor of two, due to the uncertainty in the cross sections. In a practical application, where non-uniform spatial distributions of photon and neutron spectra play a role, we estimate the global uncertainty to be at least an order of magnitude.

Some of the limitations of Swanson's technique have been mitigated by a modification recently developed at $\mathrm{CERN}^{(34)}$ in connection with the planned decommissioning of LEP $^{(4)}$. While Swanson's data refer to radionuclides produced by an electron beam of unit power incident on an infinite slab, Silari and Ulrici have compiled similar data for materials irradiated by secondary particles generated by nearby unit power beam losses in machine components. The data have been obtained both experimentally and by a new technique provided by the FLUKA Monte Carlo code, which will be described below. As an example, conversion coefficients from unit lost beam power to induced specific activity at saturation are given for stainless steel in Table $\mathbf{1 .}$ These conversion coefficients were obtained by exposing the samples at about $20 \mathrm{~cm}$ from an aluminium beam dump bombarded by $92 \mathrm{GeV}$ and $94.5 \mathrm{GeV}$ electrons or positrons.

Table 1 Conversion coefficients from average beam power (watt) to induced specific radioactivity at saturation $\mathrm{A}_{\mathrm{S}}(\mathrm{Bq} / \mathrm{g})$ for radionuclides produced in stainless steel.

\begin{tabular}{|c|c|c|c|}
\hline $\begin{array}{l}\text { Radio- } \\
\text { nuclide }\end{array}$ & $\mathrm{T}_{1 / 2}$ & $\begin{array}{l}\text { Possible } \\
\text { production } \\
\text { reactions }\end{array}$ & $\begin{array}{l}\mathrm{A}_{\mathrm{S}} \text { at saturation } \\
(\mathrm{Bq} / \mathrm{g} \text { per watt) }\end{array}$ \\
\hline${ }^{3} \mathrm{H}^{(\#)}$ & $12.3 \mathrm{y}$ & Spallation & $3.910^{-1}$ \\
\hline${ }^{22} \mathrm{Na}$ & $2.6 \mathrm{y}$ & Spallation & $8.810^{-2}$ \\
\hline${ }^{46} \mathrm{Sc}$ & $83.8 \mathrm{~d}$ & $\begin{array}{c}\text { Spallation } \\
{ }^{47} \mathrm{Ti}(\gamma, \mathrm{p}) \\
{ }^{48} \mathrm{Ti}(\gamma, \mathrm{pn})\end{array}$ & $3.910^{-1}$ \\
\hline${ }^{54} \mathrm{Mn}$ & $312.2 \mathrm{~d}$ & $\begin{array}{c}{ }^{55} \mathrm{Mn}(\gamma, \mathrm{n}) \\
{ }^{56} \mathrm{Fe}(\gamma, \mathrm{pn}) \\
{ }^{54} \mathrm{Cr}(\mathrm{p}, \mathrm{n}) \\
\end{array}$ & 10.3 \\
\hline${ }^{56} \mathrm{Co}$ & $77.7 \mathrm{~d}$ & $\begin{array}{c}{ }^{58} \mathrm{Ni}(\gamma, \mathrm{pn}) \\
{ }^{56} \mathrm{Fe}(\mathrm{p}, \mathrm{n}) \\
{ }^{57} \mathrm{Fe}(\mathrm{p}, 2 \mathrm{n}) \\
{ }^{59} \mathrm{Co}(\gamma, 3 \mathrm{n})\end{array}$ & 1.4 \\
\hline${ }^{57} \mathrm{Co}$ & $271.8 \mathrm{~d}$ & $\begin{array}{c}{ }^{58} \mathrm{Ni}(\gamma, \mathrm{p}) \\
{ }^{57} \mathrm{Fe}(\mathrm{p}, \mathrm{n}) \\
{ }^{59} \mathrm{Co}(\gamma, 2 \mathrm{n})\end{array}$ & 8.3 \\
\hline${ }^{58} \mathrm{Co}$ & $70.9 \mathrm{~d}$ & $\begin{array}{c}{ }^{60} \mathrm{Ni}(\gamma, \mathrm{pn}) \\
{ }^{59} \mathrm{Co}(\gamma, \mathrm{n}) \\
{ }^{58} \mathrm{Fe}(\mathrm{p}, \mathrm{n})\end{array}$ & 7.7 \\
\hline${ }^{60} \mathrm{Co}$ & $5.27 \mathrm{y}$ & $\begin{array}{l}{ }^{61} \mathrm{Ni}(\gamma, \mathrm{p}) \\
{ }^{63} \mathrm{Cu}(\gamma, 2 \mathrm{pn})\end{array}$ & $5.810^{-1}$ \\
\hline${ }^{85} \mathrm{Sr}$ & $64.5 \mathrm{~d}$ & ${ }^{84} \operatorname{Sr}(n, \gamma)$ & $3.010^{-1}$ \\
\hline${ }^{88} \mathrm{Y}$ & $107 \mathrm{~d}$ & ${ }^{89} \mathrm{Y}(\gamma, \mathrm{n})$ & $3.410^{-2}$ \\
\hline${ }^{88} \mathrm{Zr}$ & $83.4 \mathrm{~d}$ & ${ }^{90} \mathrm{Zr}(\gamma, 2 \mathrm{n})$ & $3.110^{-2}$ \\
\hline
\end{tabular}

(\#) values for ${ }^{3} \mathrm{H}$ are estimated from the data of ${ }^{7} \mathrm{Be}$

\section{A novel approach with FLUKA}

All the techniques reported above give results with uncertainty factors not better than 2 and in some cases of the order of 10. This has been found generally acceptable for practical purposes, because the activities around electron accelerators are usually low, although the uncertainty is not always on the conservative side. Each of the conventional methods, however, has some further drawback: they predict only part of the produced radionuclides, or lack capability to predict distribution of radioactivity in space, or require a complete cross section library which at the moment is not yet available.

The latest version of the FLUKA code $e^{(35,36)}$ opens a new way by offering at the same time a rich choice of possibilities. These allow the user to upgrade some of the conventional techniques by overcoming some of their limitations, or to predict directly the density of individual residual nuclei.

\section{Star density}

Dose rate from induced radioactivity at CERN proton accelerators has been predicted for many years by an 
empirical technique based on star density. "Stars" are arbitrarily defined as inelastic interactions, or spallations, by hadrons of energy larger than $50 \mathrm{MeV}$ and have been for a long time the only quantity predicted by high-energy hadron transport codes. The original hypothesis of a probable simple proportionality between star density and induced radioactivity, with proportionality factor dependent on material $^{(37)}$, was based on the observed constant asymptotic value of the hadron inelastic cross section and on an assumed equilibrium between the fluence of star-producing highenergy hadrons and that of other particles contributing to activation (neutrons of less than $50 \mathrm{MeV}$ ). Actually, the supposed equilibrium exists only outside thick shielding, but experience has shown that the contribution of low energy particles is generally small compared to that of starproducing hadrons. Therefore, the proportionality factors were established experimentally by measuring the gamma dose rate at the surface of small blocks of material directly irradiated by a proton beam ${ }^{(38,39)}$. It is considered that these so-called "omega-factors" are valid within approximately a factor of 3 .

Another common use of star densities obtained by Monte Carlo is to estimate individual radionuclide production by scaling the number of stars with the ratio of partial to total inelastic cross section.

These techniques cannot be extended directly to electron accelerators, where most of the induced radioactivity is due to GDR interactions and not to stars. However, outside thick shielding, where high-energy hadrons dominate, an extension is possible and a first attempt was done by Höfert et al. ${ }^{(17)}$ with the 1982 version of the FLUKA code where a first implementation of photonuclear interactions above $800 \mathrm{MeV}$ had been made. More recently, Nelson et al. ${ }^{(21)}$ have used several techniques to estimate tritium production in groundwater, among which the calculation of star density in soil with the last version of the FLUKA code.

\section{FLUKA as a source of data for conventional techniques}

Photonuclear reactions have been fully implemented in the latest version of FLUKA, from the GDR region to at least $20 \mathrm{TeV}$, giving the code the capability to handle the whole joint electromagnetic and hadronic cascade at any energy. All kinds of secondaries are generated and tracked even in very complex geometries and it is possible to calculate accurately the differential fluence of any particle (whether as tracklength, boundary crossing or collision density) in any requested region of space. Traditional techniques based on track-length estimation can therefore be applied with improved spatial resolution to the detailed fluences calculated by $\operatorname{FLUKA}^{(40)}$.

Total photon track-length above 5 or $10 \mathrm{MeV}$ is also a good candidate for a simple activity estimator for GDR interactions similar to what star density is for high-energy interactions. It would be interesting to test experimentally the performance of some combination of the two estimators.

FLUKA can be used also as a simple electron-photon transport code to estimate the partition of deposited energy among various machine or shielding components, and the result can be used to estimate activity according to the original or modified Swanson's technique.

\section{Residual nuclei}

The possibility of scoring directly residual nuclei produced in inelastic interactions has been available in FLUKA and in other hadron transport codes for some time. Although this feature can still be improved (the lack of a fragmentation model does not allow to predict nuclides with mass number very far from that of the parent nucleus well), it has already been used with success to predict activation from proton beams ${ }^{(41)}$.

Only recently attempts have been made at calculating residual nuclei produced in an electromagnetic cascade. At SLAC, the benchmark experiment of Sato et al. ${ }^{(33)}$ has been simulated, where thin $\mathrm{Al}, \mathrm{Fe}, \mathrm{Cu}$ and $\mathrm{Nb}$ foils were irradiated with a $2.5 \mathrm{GeV}$ electron beam at different depths inside a thick copper target. The detailed results will be reported elsewhere, but a summary of the results is given in Fig. 2 .

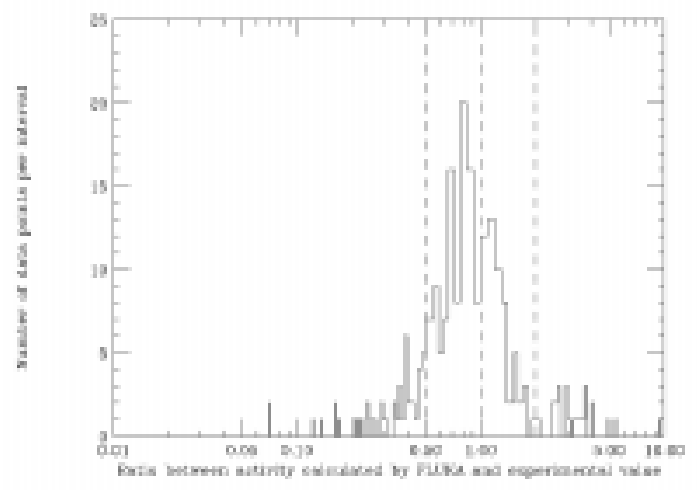

Fig. 2 Distribution of ratios between FLUKA predictions and measured activities in the experiment of Sato et $\mathrm{al}^{(33)}$. The dashed lines indicate a factor 2 about the measured value. $69 \%$ of the points are contained in the corresponding band.

Only results with a standard deviation better than $30 \%$ have been reported in the figure (it was difficult to accumulate good statistics when scoring residual nuclei in the extremely thin layers used in the experiment). Only one nuclide was completely absent in the FLUKA results $\left({ }^{24} \mathrm{Na}\right.$ in $\mathrm{Fe})$, but on the other hand several radioactive nuclides were predicted which were not measured in the experiment $\left({ }^{26} \mathrm{Al}\right.$, ${ }^{45} \mathrm{Ti},{ }^{55} \mathrm{Fe},{ }^{64} \mathrm{Cu}$, etc.). Other nuclides for which the FLUKA predictions are not so good are ${ }^{83} \mathrm{Sr}$ and ${ }^{86} \mathrm{Zr}$ in $\mathrm{Nb}$ (overestimated by factor 3 to 4) and ${ }^{84} \mathrm{Rb}$ in $\mathrm{Nb}$ (underestimated by a factor 14). About $69 \%$ of the data, reported in Fig. 2, show an agreement within a factor of 2 between calculated and measured values. As a comparison, the approach based on scaling Swanson's data with absorbed energy results in only $36 \%$ of the data points agreeing within a factor of 2, and $66 \%$ within a factor of 5 . The best FLUKA results are for ${ }^{18} \mathrm{~F}$ in aluminium, ${ }^{44} \mathrm{Sc},{ }^{48} \mathrm{~V},{ }^{52} \mathrm{Fe},{ }^{51} \mathrm{Cr},{ }^{54} \mathrm{Mn}$ in iron, ${ }^{52} \mathrm{Mn},{ }^{57} \mathrm{Co},{ }^{58} \mathrm{Co},{ }^{59} \mathrm{Fe}$ in copper and ${ }^{88} \mathrm{Zr},{ }^{89} \mathrm{Zr},{ }^{88} \mathrm{Nb}$, ${ }^{90} \mathrm{Nb}$ in niobium.

Another simulation concerned a soil sample exposed inside a tunnel near a thick dump irradiated for several weeks 
by a $50 \mathrm{GeV}$ beam. Since the chemical composition of the sample and the number of dumped electrons were only approximately known, the comparison with experimental values has less value than in the previous case. It is interesting anyway to find that the calculated yields of ${ }^{7} \mathrm{Be}$, ${ }^{22} \mathrm{Na},{ }^{48} \mathrm{~V},{ }^{51} \mathrm{Cr},{ }^{54} \mathrm{Mn},{ }^{59} \mathrm{Fe}$ and ${ }^{58} \mathrm{Co}$ agreed with the measured values within a factor of 2 , while ${ }^{3} \mathrm{H}$ was underestimated by a factor of $10^{(42)}$. However, using the star density and a reported factor of $0.09{ }^{3} \mathrm{H}$ nuclei per FLUKA star $^{(43)}$ one would still find a value 3 times too low, which seems to point to a problem in the experimental determination of tritium in the sample.

While the overall accuracy of the above results is not worse than that claimed by most established techniques, it is interesting to point out that the FLUKA yields of residual radioactive nuclei include several ones that are generally not predicted otherwise $\left({ }^{14} \mathrm{C},{ }^{55} \mathrm{Fe}\right.$, etc. $)$.

A third example is the assessment of low level induced radioactivity in the various materials constituting the LEP components, namely aluminium (vacuum chambers, dipole excitation bars), lead (shielding around most of the vacuum chamber), stainless steel (vacuum chambers, vacuum valves, bellows, etc.), iron (quadrupoles), iron-laminated concrete (dipoles) and copper (RF cavities, coils, vacuum joints, etc.). Samples of these materials were irradiated on the electron and positron dumps during the entire operation of the collider at $92 \mathrm{GeV}$ in 1997 and at $94.5 \mathrm{GeV}$ in 1998. The experimental results were compared with detailed FLUKA simulations of the experiment ${ }^{(44)}$. Both the experimental results and the Monte Carlo predictions were normalised to the average dumped beam intensity. The specific activity was obtained by multiplying the residual nuclei output from FLUKA by the average beam intensity and dividing by the mass of the sample. As an example, the results for stainless steel are given in Table 2. From the comparison between experiment and simulations, a number of conclusions could be drawn. First of all, it was found that FLUKA can predict most of the induced radionuclides in the five materials; in about $2 / 3$ of the cases the Monte Carlo results agreed with the experimental data within a factor of two and some of them even better. As stated above, FLUKA could not predict with reasonable accuracy the production of residual nuclides with atomic and mass numbers far from those of the target nuclides (i.e., spallation products such as ${ }^{7} \mathrm{Be}$ in aluminium and ${ }^{56} \mathrm{Co}$ in lead). However, the largest discrepancy was found for ${ }^{52} \mathrm{Mn}$, for which the calculated activity was about 4-5 times larger than that determined experimentally. In the experiment of Sato et al. ${ }^{(33)}$ the discrepancy for this same nuclide was a factor 2-3.

Neutrons were not followed down to thermal energy because of the long computing time required. Thus, the contribution to induced radioactivity due to thermal neutron capture was not taken into account in the calculations. By an examination of the sample compositions and induced radionuclides, it turned out that only two radionuclides might have been underestimated because of lack of contribution from thermal neutrons: ${ }^{59} \mathrm{Fe}$ in stainless steel and in ironconcrete, and ${ }^{51} \mathrm{Cr}$ in stainless steel. These nuclides are produced by ${ }^{58} \mathrm{Fe}(\mathrm{n}, \gamma){ }^{59} \mathrm{Fe}$ (cross section of $1.15 \mathrm{~b}$ ) and
${ }^{50} \mathrm{Cr}(\mathrm{n}, \gamma){ }^{51} \mathrm{Cr} \quad$ (cross section of 15.9 b), respectively. However, it was found that the latter had been predicted with good accuracy, which means that ${ }^{51} \mathrm{Cr}$ is produced mainly by interaction of high-energy neutrons rather than by thermal neutrons. Neglecting the thermal neutron reactions had only some relevance for the production of ${ }^{59} \mathrm{Fe}$ in stainless steel and iron-concrete, which is underestimated by a factor 3-5. In all other cases thermal neutrons did not contribute much to the induced radioactivity.

Table 2 Saturated specific activity of the radionuclides detected in a stainless steel sample, placed on the electron beam dump, compared with the FLUKA calculations. SD is the standard deviation. Ratio is the FLUKA/experiment ratio.

\begin{tabular}{|c|c|c|c|c|c|}
\hline \multirow{2}{*}{$\begin{array}{l}\text { Radio- } \\
\text { nuclide }\end{array}$} & \multirow{2}{*}{$\mathrm{T}_{1 / 2}$} & \multicolumn{3}{|c|}{$\begin{array}{c}\text { Specific Activity } \\
\text { (Bq/g) }\end{array}$} & \multirow{2}{*}{ Ratio } \\
\cline { 3 - 5 } & & Exp. & FLUKA & SD (\%) & \\
\hline${ }^{46} \mathrm{Sc}$ & $83.8 \mathrm{~d}$ & 0.13 & 0.065 & 12 & 0.5 \\
\hline${ }^{48} \mathrm{~V}$ & $15.97 \mathrm{~d}$ & 0.31 & 0.52 & 7 & 1.7 \\
\hline${ }^{51} \mathrm{Cr}$ & $27.7 \mathrm{~d}$ & 4.12 & 2.7 & 5 & 0.65 \\
\hline${ }^{52} \mathrm{Mn}$ & $5.6 \mathrm{~d}$ & 0.17 & 0.74 & 6 & 4.3 \\
\hline${ }^{54} \mathrm{Mn}$ & $312.2 \mathrm{~d}$ & 3.54 & 2.9 & 2 & 0.82 \\
\hline${ }^{59} \mathrm{Fe}$ & $44.5 \mathrm{~d}$ & 0.028 & 0.0088 & 27 & 0.31 \\
\hline${ }^{56} \mathrm{Co}$ & $77.7 \mathrm{~d}$ & 0.29 & 0.46 & 7 & 1.6 \\
\hline${ }^{57} \mathrm{Co}$ & $271.8 \mathrm{~d}$ & 1.3 & 1.1 & 4 & 0.85 \\
\hline${ }^{58} \mathrm{Co}$ & $70.9 \mathrm{~d}$ & 2.65 & 1.4 & 3 & 0.52 \\
\hline${ }^{60} \mathrm{Co}$ & $5.27 \mathrm{y}$ & 0.18 & 0.085 & 21 & 0.47 \\
\hline${ }^{95} \mathrm{Nb}$ & $34.9 \mathrm{~d}$ & 0.038 & 0.013 & 27 & 0.34 \\
\hline
\end{tabular}

\section{Conclusions}

Comparisons made at CERN and SLAC show that there is a satisfactory agreement between the results derived by conventional techniques and those obtained by exploiting the new options offered by FLUKA. No existing method is perfect, but the integrated approach now allowed by FLUKA is better than most of the other techniques.

Part of this work was supported by the Department of Energy under contract DE-A-03-76SF00515.

\section{REFERENCES}

(1) Schönbacher, H., Tavlet, M.: Radiation protection aspects of the decommissioning of the CERN Intersecting Storage Ring; CERN TIS-RP/198/CF (1987).

(2) Schönbacher, H., Tavlet, M.: Démontage des ISR. Activités du service de radioprotection; CERN TIS-RP/IR/85-03 (1985).

(3) McCall, R.C., et al.: U.S. Department of Energy, Health physics manual of good practices for accelerator facilities; SLAC Report 327 (1988).

(4) Fassò, A., et al.: Induced radioactivity calculations in view of the large electron positron collider decommissioning; these proceedings.

(5) Dietrich, S.D., Berman, B.L.: At. Data Nucl. Data Tables 38, 199 (1988).

(6) CDFE: Center for photonuclear experiments data http://depni.npi.msu.su/cdfe/ 
(7) Rudstam, G.: Z. Naturforsch. 21a, 1027 (1966).

(8) Jonsson, G.G., Lindgren, K.: Phys. Scr., 15, 308 (1977).

(9) Fassò, A., et al.: Production of radioactive gas in LEP; Soil and water activation; Sealed and contained radioactivity; in "The radiological impact of the LEP project on the environment", Ed. K. Goebel, CERN, 81-08 (1981).

(10) Goebel, K.: Radioecological considerations for operating particle accelerators; CERN 79-09 (1979).

(11) DeStaebler, H.: Photon-induced residual activity; SLAC TN63-92 (1963).

(12) Thomas, R.H.: The radiological impact of high-energy accelerators on the environment; KEK-78-20 (1978).

(13) Weingarten, W.: Superconducting cavities; CAS RF engineering for particle accelerators, CERN 92-03, p. 318 (1992).

(14) Lengeler, H.: X-ray radiation from a module of 4 superconducting cavities; CERN Technical Note CERN/EF/4420H/H/ed (1989).

(15) Lengeler, H.: Radiation protection of measuring set-ups for LEP 4-cell cavity; CERN Technical Note CERN/EF/4370H/HL/ed (1989).

(16) Silari, M., et al:: Radiation produced by the LEP superconducting RF cavities; CERN TIS-99-003-RP-PP (1999); NIM A (in press).

(17) Höfert, M., Jin, S., Stapleton, G.: Considerations of the use of the EGS/FLUKA Monte Carlo code to determine the activity of $\mathrm{H}-3$ and Na-22 in the groundwater; in ref. 25, p. A4-11.

(18) Höfert, M., Sievers, P.: Radiological problems at high-energy, high intensity electron-positron converters; CERN TIS-RP/212 (1988).

(19) Nelson, W.R.: Radioactive ground water produced in the vicinity of beam dumps; SLAC-TN-65-16 (1965).

(20) Stapleton, G.: The production of radionuclides in groundwater; CEBAF TN-0062 (1987).

(21) Nelson, W.R., et al.: Estimate of tritium production in ground water near SLC beam dumps; SLAC Note RP-98-2/Rev (1998).

(22) Racky, B., et al.: Radiation environment of the linear collider TESLA; Proc. 4th Workshop on simulating accelerator environments (SARE4), 14-16 September 1998, Knoxville (USA), p. 303 (1998).

(23) Kosako, T., Nakamura, T.: Health Phys. 43, 3 (1982)

(24) Fassò, A., Ferrari, A., Sala, P.R.: Total giant resonance photonuclear cross sections for light nuclei: a database for the FLUKA Monte Carlo transport code, Proc. 3rd Specialists' Meeting on Shielding Aspects of Accelerators, Targets and Irradiation Facilities (SATIF3), Tohoku University, Sendai, Japan, 12-13 May 1997, OECD-NEA, p. 61 (1998).

(25) Review of the radiological aspects of the conceptual design report for the CEBAF end stations and switchyard, CEBAF TN-0174 (several collected Technical Notes) (1989).

(26) Leuschner, A., Stevenson, G.R.: Studies of the radioactivity induced by synchrotron radiation in LEP dipoles; CERN TISRP/IR/99-12 (1999).

(27) Swanson, P.: "Radiological safety aspects of the operation of electron linear accelerators"; Technical Report Series n. 188, ed. IAEA, Vienna (1979).

(28) Huntzinger, C.J., Holmes, J.A.: Production and control of activation products at an electron linear accelerator complex; Health Physics of Radiation Generating Machines, Proc. 20th midyear topical Symposium of the Health Physics Society, Reno, Nevada, 8-12 February 1987, p. 539 (1987).

(29) Rossi, B.: High-Energy Particles, ch. 5, Prentice-Hall, Englewood Cliffs, NJ (1952).
(30) Rossi, B., Greisen, K.: Cosmic-ray theory; Rev. Mod. Phys. 13, 240 (1941).

(31) Swanson, W.P.: Health Phys. 28, 495 (1975).

(32) Nelson, W.R., Hirayama, H., Rogers, D.W.O.: The EGS4 code system; SLAC-265 (1985).

(33) Sato, T., et al.: Nucl. Instr. Meth., A401, 476 (1997).

(34) Silari, M., Ulrici, L.: Reference values for estimating induced radioactivity in LEP; CERN TIS-RP/IR/99-17 (1999).

(35) Fassò, A., et al.: New developments in FLUKA modelling of hadronic and EM interactions; Proc. Third Workshop on Simulating Accelerator Radiation Environments (SARE3), KEK, Tsukuba, Japan, 7-9 May 1997, Ed. H. Hirayama, KEK Proceedings 97-5, p. 32 (1997).

(36) Ferrari, A., Rancati, T., Sala, P.R.: Fluka applications in highenergy problems: from LHC to ICARUS and atmospheric showers; Proc. Third Workshop on Simulating Accelerator Radiation Environments (SARE3), KEK, Tsukuba, Japan, 7-9 May 1997, Ed. H. Hirayama, KEK Proceedings 97-5, p. 165 (1997).

(37) Ranft, J., Goebel, K.: Estimation of induced radioactivity around high-energy accelerators from hadronic cascade star densities obtained from Monte Carlo calculations; CERN HP70-92 (1970).

(38) Höfert, M., et al.: The prediction of radiation levels from induced radioactivity: discussion of an internal dump target facility in the PS; CERN Report DI/HP/185 (1975).

(39) Höfert, M., Bonifas, A.: Measurement of radiation parameters for the prediction of dose-rates from induced radioactivity; CERN Report HP-75-148 (1975).

(40) Fassò, A., Ferrari, A., Sala, P.R.: Designing accelerator shielding with FLUKA; Proc. $8^{\text {th }}$ International Conference on Radiation Shielding, 24-28 April 1994, Arlington (USA), American Nuclear Society, p. 643 (1994).

(41) Ferrari, A., Sala, P.R.: Intermediate and high energy models in FLUKA: improvements, benchmarks and application, Proc. International Conference on Nuclear Data for Science and Technology, NDST-97, International Centre for Theoretical Physics, Miramare-Trieste, Italy, published by Italian Physical Society, Conference Proceedings, G. Reffo, A. Ventura and C. Grandi eds., ISBN 88-7794-114-6, Bologna, Part I, p. 247 (1997).

(42) Liu, J.C., et al.: Environmental impact from accelerator operation at SLAC; SLAC-PUB-8053 (1999).

(43) Dinter, H., Tesch, K.: Investigation of the protection of the environment against radiation at the storage ring HERA; DESY HERA 80/04 (1980), (cited in (17)).

(44) Silari, M., Ulrici, L., Ye, S.: On the estimation of low levels of induced radioactivity in LEP; CERN TIS-RP/IR/99-13 (1999). 